\title{
Insertion and deletion mutations in the dinucleotide repeat region of the Norrie disease gene in patients with advanced retinopathy of prematurity
}

\begin{abstract}
Retinopathy of prematurity (ROP) is a leading cause of blindness in premature children. It is a multifactorial disorder which causes fibrovascular tissue changes that affect the retina in low birth-weight and short gestational age infants. To determine the prevalence of Norrie disease $(N D)$ gene mutations, clinical examination and molecular genetic analyses were performed in 100 pre-term babies of different ethnic backgrounds who developed advanced ROP. The leukocyte DNA was extracted, amplified by the polymerase chain reaction (PCR), and analyzed by singlestrand conformation polymorphism (SSCP), G/T and C/A scanning, and by DNA sequencing. All three exons, including splice sites and the $3^{\prime}$-untranslated region, were screened. Of the 100 patients analyzed, 2 patients with advanced ROP showed a mobility shift in the DNA. In 1 patient, this mobility shift was caused by the insertion of an additional 12-bp CT repeat in exon 1, and in the second patient, there was a 14-bp deletion in the same exon of the $N D$ gene, as evidenced by direct sequencing of the amplified products. Similar analyses of exons 2 and 3 and the 3 'untranslated region failed to detect additional mutations in the gene. None of the 130 normal, unrelated controls revealed similar changes. Taking into account the above results, as well as those of other studies, it appears that the $N D$ gene mutations can account for $3 \%$ of cases of advanced ROP. Although the $N D$ gene is not frequently involved in advanced ROP, the present large-scale study further supports the hypothesis that genetic influences may play an important role in the development of severe ROP in some premature infants.
\end{abstract}

M. Hiraoka $\cdot$ B.S. Shastry $(\square)$

Department of Biological Sciences, Oakland University, Rochester, MI 48309, USA

Tel. +1-248-370-2994; Fax +1-248-370-4225

e-mail: Barkur@aol.com

D.M. Berinstein

Department of Ophthalmology and Visual Sciences, Yale University School of Medicine, New Haven, CT, USA

M.T. Trese

William Beaumont Hospital, Royal Oak, MI, USA
Key words Mutations $\cdot$ Sequencing $\cdot$ Norrie disease $\cdot \mathrm{X}$ linked $\cdot$ Retinopathy

\section{Introduction}

Retinopathy of prematurity (ROP) is a well known cause of visual impairment in premature children. It is a fibrovascular tissue change that affects the retina in low birth-weight and short gestational age infants. The clinical manifestations of advanced ROP are similar to those of familial exudative vitreoretinopathy (FEVR) (Phelps 1993). While the etiology of ROP is currently not known, many causative factors have been proposed. Recently, a role of vascular endothelial growth factor (VEGF) has been suggested for ROP, but this still failed to answer the question of why only particular children are the targets of advanced ROP. Additionally, early exposure to excessive light is not found to be a factor in the development of ROP (Reynolds et al. 1998). Despite advances in our understanding and management of ROP, each year many children will experience total blindness caused by ROP. It is unclear at present why ROP, in a subset of infants with low birth weight, progresses to a severe stage, despite timely intervention, whereas in other infants with similar clinical characteristics, ROP regresses spontaneously. Although no biochemical defects have been identified in animal and human studies, this unpredictability of ROP could be caused by genetic influences (Shastry et al. 1997; Buffenn et al. 1999).

\section{Subjects and methods}

Patients

To further investigate the incidence and role of genetic factors in advanced ROP, we studied three types of populations: group A was composed of 100 patients with advanced ROP, including three pairs of dizygotic and two pairs of monozygotic twins; group B was composed of 6 patients 
with stage 3 ROP that had regressed without treatment; and group $\mathrm{C}$ was composed of 130 unrelated, randomly selected normal control subjects. Patients with a history of bleeding disorder, anemia, or ocular disorders unrelated to ROP were excluded from these groups. All the patients in group A had clinical findings typical of advanced ROP (stages 4B and 5), and all the patients in group B showed typical signs of regressed ROP. The birth weight and gestational age of the pre-term babies ranged from 600 to $1300 \mathrm{~g}$ (mean, $882 \mathrm{~g}$ ) and 23 to 30 weeks (mean, 26 weeks), respectively. This patient set contained $63 \%$ males and $37 \%$ females $(98 \%$ Caucasians and 2\% African-Americans). After Institutional Review Board (IRB) approval and informed consent were obtained, peripheral blood samples from all patients were collected. For the purpose of mutational analysis, we selected the Norrie disease $(N D)$ gene as a candidate gene possibly causing severe ROP, based on our own, as well as other studies (Shastry et al. 1997; Buffenn et al. 1999).

\section{Methods}

The leukocyte DNA was extracted, amplified by the polymerase chain reaction (PCR), and analyzed by single-strand conformational polymorphism (SSCP), G/T and C/A scanning, and by direct DNA sequencing. All three exons, including the splice sites and the $3^{\prime}$ untranslated (460-bp) region, were screened using primers designed to amplify exons of the $N D$ gene (Meindl et al. 1992). The PCR amplification was carried out using Applied Biosystems (Foster City, CA, USA) Taq DNA polymerase, with 30 cycles of $1.5 \mathrm{~min}$ at $94^{\circ} \mathrm{C}, 1 \mathrm{~min}$ at $60^{\circ} \mathrm{C}$, and $2 \mathrm{~min}$ at $72^{\circ} \mathrm{C}$ in the manufacturer's buffer, containing 2-4 units of polymerase, $10 \mathrm{pmol}$ each of the primer, $50 \mu \mathrm{M}$ each of four deoxynucleotides, $1.5 \mathrm{mM} \mathrm{MgCl}$, and $10 \mathrm{mM}$ tris- $\mathrm{HCl}, \mathrm{pH}$ 8.3. The PCR products were purified and directly sequenced using a cycle sequencing kit (Applied Biosystems) according to the manufacturer's instructions. The sequencing reactions were analyzed on $6 \%$ polyacrylamide gels containing $8 \mathrm{M}$ urea.

\section{Results}

In order to determine the prevalence of $N D$ gene mutations, clinical examination and molecular genetic analyses were performed in 100 children with a history of premature birth and low birth weight. The following inclusion criteria had to be met: (a) a gestational age of 30 weeks, (b) a birth weight of $1500 \mathrm{~g}$ or less, (c) a postconceptual age of at least 12 months, and (d) retinal findings consistent with ROP, including time of onset and tempo. All babies had developed stage 4B/5 ROP. The $N D$ gene contains three exons and the protein coding sequence is confined within exons 2 and 3 , with the first exon untranslated. A portion of the analysis of exon 1 is shown in Fig. 1A,B. Of the 100 patients analyzed, two male patients showed a mobility shift in the DNA. In one patient, this mobility shift was caused by the insertion of additional CT repeats (12bp) in the dinucle-
A

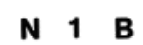

5

10

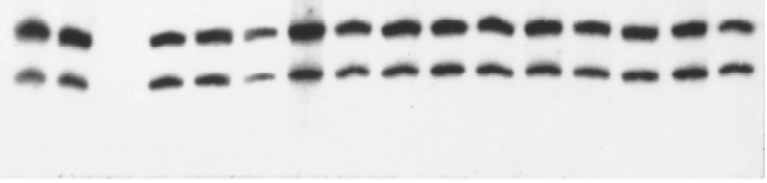

B

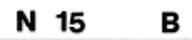

20

25

28

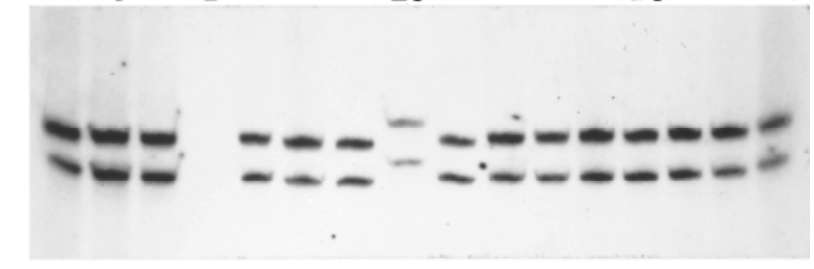

C

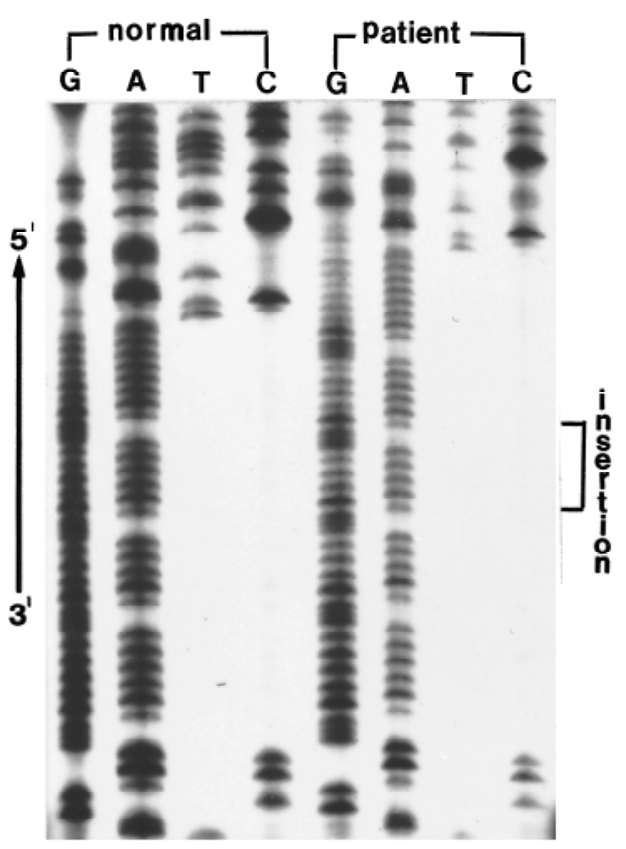

Fig. 1A-C. Mutational analysis of the Norrie disease $(N D)$ gene. A and B Show single-strand conformational polymorphism (SSCP) analysis of the $N D$ gene exon 1 in 28 patients with advanced retinopathy of prematurity (ROP). Mobility shift was observed in patient no. 20. $N$ and $B$ refer to normal and blank lanes, respectively. C Shows the nucleotide sequence of the mutant part of the $N D$ gene exon 1 in ROP patient no. 20. The sequence change is an insertion of additional CT repeats $(12 \mathrm{bp})$ in the dinucleotide repeat, region, which was not seen in 130 normal healthy individuals

otide repeat region, as evidenced by direct sequencing (Fig. 1C) of the amplified product (the patient's mother, unfortunately, declined to be tested). In the second patient, similar analyses showed a 14-bp deletion in the same exon (Fig. 2) of the $N D$ gene. To our knowledge, this deletion mutation has not been previously reported, and, hence, represents a novel mutation in the $N D$ gene. None of the other babies, including those who had stage 3 ROP but regressed, and the 130 unrelated normal controls, revealed similar changes. Similar analyses of exons 2 and 3 and the 3'- 


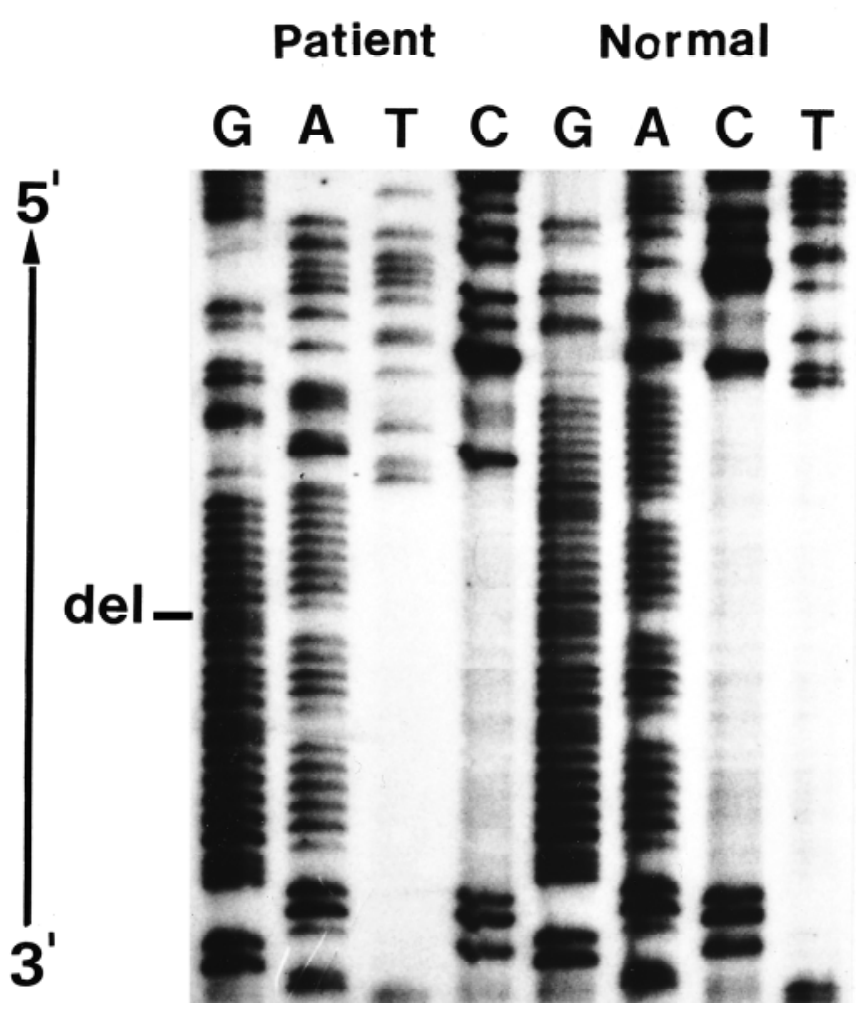

Fig. 2. The nucleotide sequence of the mutant part of the $N D$ gene exon 1 in an ROP patient. The sequence change is a 14-bp deletion in the CT repeat region. This mutation was not detected in 130 normal healthy controls

untranslated region failed to detect any additional changes in the gene. Because (i) an expansion within the CT repeat has been shown to be associated with patients expressing the full ND phenotype (Schuback et al. 1995); (ii) no other mutations have been identified throughout the coding and noncoding regions of the $N D$ gene; (iii) more than 130 normal controls studied did not show a similar repeat expansion; and (iv) the CT repeat region has been shown to control the expression of the gene (Kenyon and Criag 1999), it is very likely that the dinucleotide repeat expansion and deletion identified in the patients have some relationship to advanced ROP. It is possible that such an insertion or deletion alters the regulation of gene expression, message stability, or translatability of mRNAs, affecting the amount of protein produced. In $N D$ and $F E V R$, however, most of the mutations reported to date are missense mutations, which may change the structure and function of the protein. At present we are also unable to comment on the effect of prematurity on this mutation.

\section{Discussion}

ROP is becoming a major cause of blindness among children in middle-income countries. Despite advances in our understanding and management of ROP, it is unclear at present why ROP in a subset of infants with low birth weight progresses to a severe stage, despite timely intervention, whereas, in other infants with similar clinical characteristics, ROP regresses spontaneously. We hypothesized that this unpredictability could be caused by genetic influences. In order to address this question, we undertook the present large-scale study of the $N D$ gene, which has previously been shown to be involved in some cases of advanced ROP (Shastry et al. 1997). Our extensive analyses, together with the results of other studies (Shastry et al. 1997; Buffenn et al. 1999), suggest that the $N D$ gene mutations can account for $3 \%$ of cases of advanced ROP. This indicates that the $N D$ gene plays a much smaller role in ROP than was previously thought. The two patients with mutations in the $N D$ gene in this study had clinical findings typical of advanced ROP (advanced ROP develops through stages, whereas ND does not). These infants (birth weight, less than $1500 \mathrm{~g}$ ) developed threshold ROP at the postconceptional age of 34 to 36 weeks. The disease had progressed to stage $4 \mathrm{~B} / 5$ at the postconceptional age of 38 to 40 weeks, which is typical of advanced ROP.

Given the fact that ROP is a nonMendelian disease (multifactorial or common complex), it is understandable that the $N D$ gene (or a single gene) cannot be a candidate gene for all severe cases of ROP. Even in Mendelian disorders such as X-linked retinitis pigmentosa (RP) and familial breast cancer, single genes are involved in only $10 \%$ and $5 \%$ of the cases, respectively (Hardcastle et al. 1999). Multifactorial diseases such as diabetes, obesity, and Alzheimer disease have a population frequency greater than $1 \%$ and differ from single-gene diseases such as cystic fibrosis and muscular dystrophy. Even if an identical twin has a multifactorial disease, the other twin will not necessarily develop the disease. Therefore, the above finding of the $N D$ gene mutation in ROP patients (prevalence, about 10\%) is within the expected range and reasonable. Although the $N D$ gene is not frequently involved in all severe ROP cases, the present study further supports the hypothesis that some of the unpredictability of ROP could be caused by genetic influences. Several observations made in previous studies, such as (a) racial variation; (b) substantial differences in the incidence of severe ROP among different industrialized nations; (c) failure to reproduce cicatricial ROP in animal models; and (d) the case-to-case similarity of advanced ROP to a well known familial disorder, FEVR (which is associated with mutations in the $N D$ gene), support our findings. While mutations in the $N D$ gene are implicated in a small number of cases, mutations involving other proteins involved in retinal development and angiogenesis could also be associated with severe ROP.

Acknowledgments We thank the members of the families who were involved in this study. The study was supported in part by a grant from the March of Dimes Foundation.

\section{References}

Buffenn AN, Bernstein SL, Hutcheson KA (1999) Identification of a new Norrie disease gene mutation in an African-American infant 
with advanced retinopathy of prematurity. Invest Ophthalmol Vis Sci 40(Suppl):S565

Hardcastle AJ, Thiselton DL, Maldergem LV, Saha BK, Jay M, Plant C, Taylor R, Bird AC, Bhattacharya S (1999) Mutations in the RP2 gene cause disease in $10 \%$ of families with familial $\mathrm{X}$-linked retinitis pigmentosa assessed in this study. Am J Hum Genet 64:1210-1215

Kenyon JR, Criag IW (1999) Analysis of the 5'-regulatory region of the human Norrie disease gene: evidence that a non-translated CT dinucleotide repeat in exon 1 has a role in controlling expression. Gene 227:181-188

Meindl A, Berger W, Meitinger T, Van de Pool D, Achatz H, Dorner C, Haasemann M, Hellebrand H, Gal A, Cremers F, Ropers H-H (1992) Norrie disease is caused by mutations in an extracellular protein resembling C-terminal globular domain of mucin. Nat Genet 2:139-143

Phelps DL (1993) Retinopathy of prematurity. Pediatr Ophthalmol 40:705-714

Reynolds JD, Hardy RJ, Kennedy KA, Spencer R, Van Heuven WAJ, Fielder AR (1998) Lack of efficacy of light reduction in preventing retinopathy of prematurity. N Engl J Med 338:1572-1576

Schuback DE, Chen ZY, Craig IW, Breakfield XO, Sims KB (1995) Mutations in the Norrie disease gene. Hum Mutat 5:285-292

Shastry BS, Pendergast SD, Hartzer MK, Liu X, Trese MT (1997) Identification of missense mutations in the Norrie disease gene associated with the advanced retinopathy of prematurity. Arch Ophthalmol 115:651-655 